\title{
Clinical and Radiological Results of Microsurgical Posterior Lumbar Interbody Fusion and Decompression without Posterior Instrumentation for Lateral Recess Stenosis
}

\author{
Mehmet Demirayak ${ }^{1}$, Lokman Şişman ${ }^{2}$, Faik Türkmen ${ }^{3}$, Duran Efe ${ }^{4}$, \\ Oğuzhan Pekince ${ }^{3}$, Recep Gani Göncü ${ }^{1}$, Cem Sever ${ }^{5}$ \\ ${ }^{1}$ Department of Orthopaedic Surgery, Mevlana (Rumi) University Faculty of Medicine, Konya, Turkey \\ ${ }^{2}$ Department of Neurosurgery, Beyhekim State Hospital, Konya, Turkey \\ ${ }^{3}$ Department of Orthopaedic Surgery, Meram Medical School of Necmettin Erbakan University, Konya, Turkey \\ ${ }^{4}$ Department of Radiology, Mevlana (Rumi) University Faculty of Medicine, Konya, Turkey \\ ${ }^{5}$ Department of Orthopaedic Surgery, Medipol University Faculty of Medicine, Istanbul, Turkey
}

Study Design: A single-center, retrospective patient review of clinical and radiological outcomes of microsurgical posterior lumbar interbody fusion and decompression, without posterior instrumentation, for the treatment of lateral recess stenosis.

Purpose: This study documented the clinical and radiological results of microsurgical posterior lumbar interbody fusion and decompression of the lateral recess using interbody cages without posterior instrumentation for the treatment of lateral recess stenosis.

Overview of Literature: Although microsurgery has some advantages, various complications have been reported following microsurgical decompression, including cage migration, pseudoarthrosis, neurologic deficits, and persistent pain.

Methods: A total of 34 patients (13 men, 21 women), with a mean age of $56.65 \pm 9.1$ years (range, 40-77 years) confirmed spinal stability, and preoperative radiological findings of lateral recess stenosis, were included in the study. Interbody polyetheretherketone cages and auto grafts were used in all patients. Posterior instrumentation was not used because of limited resection of the posterior lumbar structures. Preoperative and postoperative radiographs, computed tomography scans, and magnetic resonance imaging were assessed and compared to images taken at the final follow-up. Functional recovery was also evaluated according to the Macnab criteria at the final follow-up.

Results: The average follow-up time was $35.05 \pm 8.65$ months (range, 24-46 months). The clinical results, operative time, intraoperative blood loss, and duration of hospital stay were similar to previously published results; the fusion rate (85.2\%) was decreased and the migration rate $(5.8 \%)$ was increased, compared with prior reports.

Conclusions: Although microsurgery has some advantages, migration and pseudoarthrosis remain challenges to achieving adequate lumbar interbody fusion.

Keywords: Microsurgical; Lateral recess stenosis; Posterior lumbar interbody fusion; Without posterior instrumentation; Cage migration

Received Jan 13, 2015; Revised Feb 16, 2015; Accepted Mar 6, 2015

Corresponding author: Mehmet Demirayak

Department of Orthopaedic Surgery, Mevlana (Rumi) University Faculty of Medicine, Meram, Konya, Turkey

Tel: +90-536-248-0492, Fax: +90-332-322-9419, E-mail: mdemirayak@mevlana.edu.tr 


\section{Introduction}

Lateral recess stenosis (LRS), a type of degenerative lumbar spinal stenosis with degenerative disc disease, is clinically characterized by radiating lower back pain and claudication when walking, sensory disturbances, muscle weakness, and urinary and defecatory obstructions in severely affected patients. Sciatic pain specifically, which was relieved during flexion and worsened with extension, is also commonly observed [1-4]. Many techniques have been used to treat degenerative lumbar spinal stenosis and LRS including traditional open decompressive surgery and posterior lumbar interbody fusion (PLIF).

Traditional open decompressive lumbar surgery for the treatment of spinal stenosis and LRS was described by Lane [5] in 1893; and Young et al. [6] first used a unilateral approach through the surgical corridor for spinal canal decompression in 1988. Traditional open surgeries, like posterolateral fusion with decompression, include total laminectomy and resection of the facet joint, as needed, combined with a pedicle screw fixation. The procedure results in a successful surgical treatment of LRS [2,7]. The advantages of simple decompression include faster pain relief and high patient satisfaction during the early postoperative period [7]. However, a pedicle screw fixation also contributes to posterolateral fusion between the intertransverse processes and the lateral aspects of the facet joints $[8,9]$. Older patients may have comorbidities such as low bone density, which increase complication rates during spinal surgery [7]. If decompression is not combined with a pedicle screw fixation, symptoms frequently recur within years [10].

PLIF, used to treat several spinal diseases, was first described by Cloward [11] in 1953. Open PLIF, involving the use of an interbody cage and posterior pedicle screw instrumentation, has been widely used for restoring disc height, decompressing the spinal nerve roots and dural sac, and segmental alignment during the treatment of LRS [12-14]. Tissue denervation and ischemia, associated with the traditional posterior approach, have been reported by many authors and cause continued postoperative symptoms $[15,16]$ PLIF, without posterior pedicle fixation to reduce these complications, can cause cage migration and iatrogenic instability $[12,17]$.

Spinal microsurgical techniques have been gaining popularity in conjunction with recent technological advances in illumination, instrumentation, and magnification [4].
The advantages of the microsurgical technique include less soft tissue damage, less intraoperative blood loss, faster postoperative pain relief, shorter hospitalizations, and faster returns to daily life and work; these advantages have been reported by many authors $[2,18,19]$. There is no need for posterior instrumentation, if the lumbar spine is shown to be stable in the patient's most recent microsurgical spine surgery. In such cases, microscopy guided resection of less than half of the medial facet, partial hemilaminotomy, and insertion of the cages may be performed through this window, thus leading to stable posterior structures $[2,16]$. However, spinal microsurgery is also associated with a steep learning curve, incomplete spinal decompression, and the need for special equipment $[2,16]$.

This study reports the single-center clinical and radiological results of microsurgical PLIF and decompression, without posterior instrumentation, as a surgical treatment option for treating LRS.

\section{Materials and Methods}

\section{Patients}

Between February 2010 and November 2012, 34 patients underwent microsurgical PLIF and decompression for LRS with a degenerative disc disease; these individuals were retrospectively reviewed as part of this study. The indication for operative treatment was the absence of a positive response to three months of conservative treatment; continued lower limb pain was the most important criterion indicating the need for the surgical treatment. The study group included 13 men and 21 women, aged 40 to 77 years (mean, $56.65 \pm 9.1$ years). This study was performed according to the principles of the Declaration of Helsinki and was approved by the Ethics Committee of the Meram Medical School of Necmettin Erbakan University (Konya, Turkey)

\section{Imaging diagnoses}

At the final follow-up, lateral hyper flexion and hyperextension radiographs, and computed tomography (CT) and magnetic resonance imaging (MRI) images were compared with preoperative radiological findings. Fusion rates, changes in disc height, and spinal canal diameters were evaluated; all radiologic assessments were performed by one radiologist. Disc height was measured at 
the midpoints of adjacent vertebral bodies. Lateral recess obliteration because of a hypertrophied facet and herniated disc, as seen in an MRI, is essential for the diagnoses of LRS. Fusion was defined as continuous bony bridging at either the intertransverse space or in and around the cages on coronal and sagittal MRI, CT, and X-ray images. The influences of artifacts resulting from materials were unremarkable.

\section{Clinical evaluation}

Functional recovery was evaluated, according to the Macnab criteria at the final follow-up. The outcomes were described as excellent (complete pain relief, complete motor recovery, return to work and daily activities); good (infrequent pain, improved main symptoms, normal muscle strength, negative nerve stress tests, tolerable light physical activities); fair (improved symptoms, with persistent back pain and sciatica during daily activities); and poor (no change in symptoms and requiring further surgery).

\section{Surgical procedure}

The PLIF procedure was performed, with the patient in the prone position and $90^{\circ}$ flexed hips, through a small, median, posterior lumbar incision, and using a bilateral approach. The surgery was performed by a neurosurgeon. The procedure included: a fluoroscopic detection of the correct surgical level, paraspinal stripping of the muscle from the spinous process, placement of a tubular retractor over the facet joint, microscopic partial hemilaminotomy, resection of less than half of the medial facet, decompression of the nerve roots at the lateral recess, removal of the ligamentum flavum, medial retraction of the dura mater with lateral retraction of the nerve root, and excision of the disc through this window, preparation of the endplates using a curette, measuring the gap with templates (size $0.5 \times 0.7-1.2 \mathrm{~cm}$ ), and an insertion of a fenestrated interbody polyetheretherketone (PEEK) cage filled with autograft material ( $2 \mathrm{~mL}$ ) obtained during surgery. The other side underwent the same surgery with another fenestrated interbody PEEK cage. The anatomic layers were closed in a routine manner.

\section{Statistics}

Clinical and radiologic results were evaluated using SPSS ver. 16.0 (SPSS Inc., Chicago, IL, USA) to conduct a repeated measures variance analyses; a two-tailed $p$-value $\leq 0.05$ was accepted as being statistically significant.

\section{Results}

In all 34 patients, the indication for surgery was LRS with degenerative disc disease. Six patients required 3-level fusions, 12 patients required 2-level fusions and 16 patients underwent single-level surgeries. Solid fusion (Fig. 1) was observed in 29 patients (85.2\%). The average disc heights were $1.27 \pm 0.59 \mathrm{~cm}, 1.34 \pm 0.68 \mathrm{~cm}$, and $1.28 \pm 0.66 \mathrm{~cm}$ when measured preoperatively, postoperatively, and at the last follow-up, respectively. The disc height increased, on average, $0.07 \pm 0.09 \mathrm{~cm}$, postoperatively; however, the difference was not significant $(p=0.198)$. At the final followup, there was no mean difference in disc height, compared with the preoperative height $(p=0.811)$. The mean spinal canal diameters were $1.32 \pm 0.69 \mathrm{~cm}, 2.90 \pm 1.57 \mathrm{~cm}$, and $2.78 \pm 1.19 \mathrm{~cm}$ when measured preoperatively, postoperatively, and at the last follow-up, respectively. The mean diameter of the patients' spinal canals increased significantly $(1.62 \pm 0.08 \mathrm{~cm})$ between the pre- and postoperative measurements $(p<0.001)$. The mean spinal canal diameter did not change significantly between the postoperative and final follow-up measurements $(p=0.274)$ (Table 1).

Clinical recoveries, as evaluated at the final follow-up using the Macnab criteria, were excellent (17 cases, 50\%), good (11 cases, $32.3 \%$ ), poor ( 3 cases, $8.8 \%$ ), or fair ( 3 cases, $8.8 \%)$.

Postoperative complications included root injury because of cage migration (two patients), superficial infection (one patient), dural tear (one patient), and nonunions (five patients). Postoperative infection was treated with $s$ (cefazolin). The root injuries, manifesting as weakness during ankle dorsiflexion and numbness, were detected in two patients who recovered spontaneously prior to their last follow-up assessment. Revision surgery was required in 1 patient because of severe cage migration, resulting in foot drop (Fig. 2). The dural sac tear was repaired intraoperatively. Most complications were conservatively treated, except for the patient with foot drop, which persisted and required surgical intervention. Continued sciatica was observed in two patients.

The mean operative time was $149.76 \pm 52.99$ minutes (range, 90-280 minutes). Patients with multi-level fusions required almost 90 minutes more surgical time than pa- 

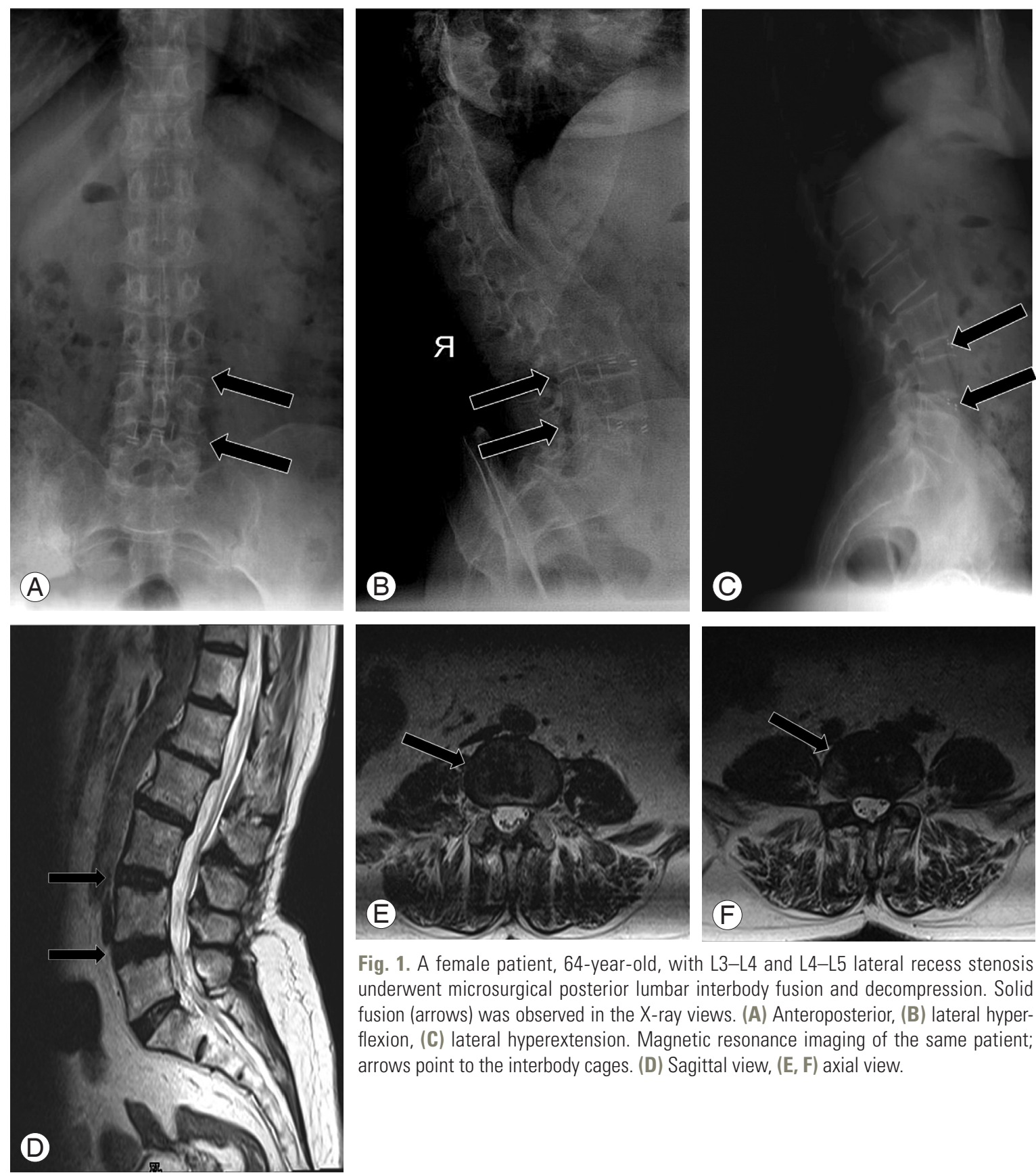

Fig. 1. A female patient, 64-year-old, with $L 3-L 4$ and $L 4-L 5$ lateral recess stenosis underwent microsurgical posterior lumbar interbody fusion and decompression. Solid fusion (arrows) was observed in the X-ray views. (A) Anteroposterior, (B) lateral hyperflexion, (C) lateral hyperextension. Magnetic resonance imaging of the same patient; arrows point to the interbody cages. (D) Sagittal view, (E, F) axial view.

Table 1. Changes in mean disc height and spinal canal diameter throughout the study period

\begin{tabular}{lccccc} 
Variable & Preoperative & Postoperative & Last follow-up & Increase & $p$-value \\
\hline Disc height $(\mathrm{cm})$ & $1.27 \pm 0.59$ & $1.34 \pm 0.68$ & $1.28 \pm 0.66$ & $0.07 \pm 0.09$ & 0.198 \\
Canal diameter $(\mathrm{cm})$ & $1.32 \pm 0.69$ & $2.78 \pm 1.19$ & $2.78 \pm 1.19$ & $1.62 \pm 0.08$ & 0.000 \\
\hline
\end{tabular}

Values are presented as mean \pm standard deviation.

tients requiring a single-level fusion. The mean blood loss was $273.38 \pm 111.84 \mathrm{~mL}$ (range, $150-500 \mathrm{~mL}$ ). Similarly, patients with multi-level fusions experienced approximately $150 \mathrm{~mL}$ more blood loss than patients with single- 

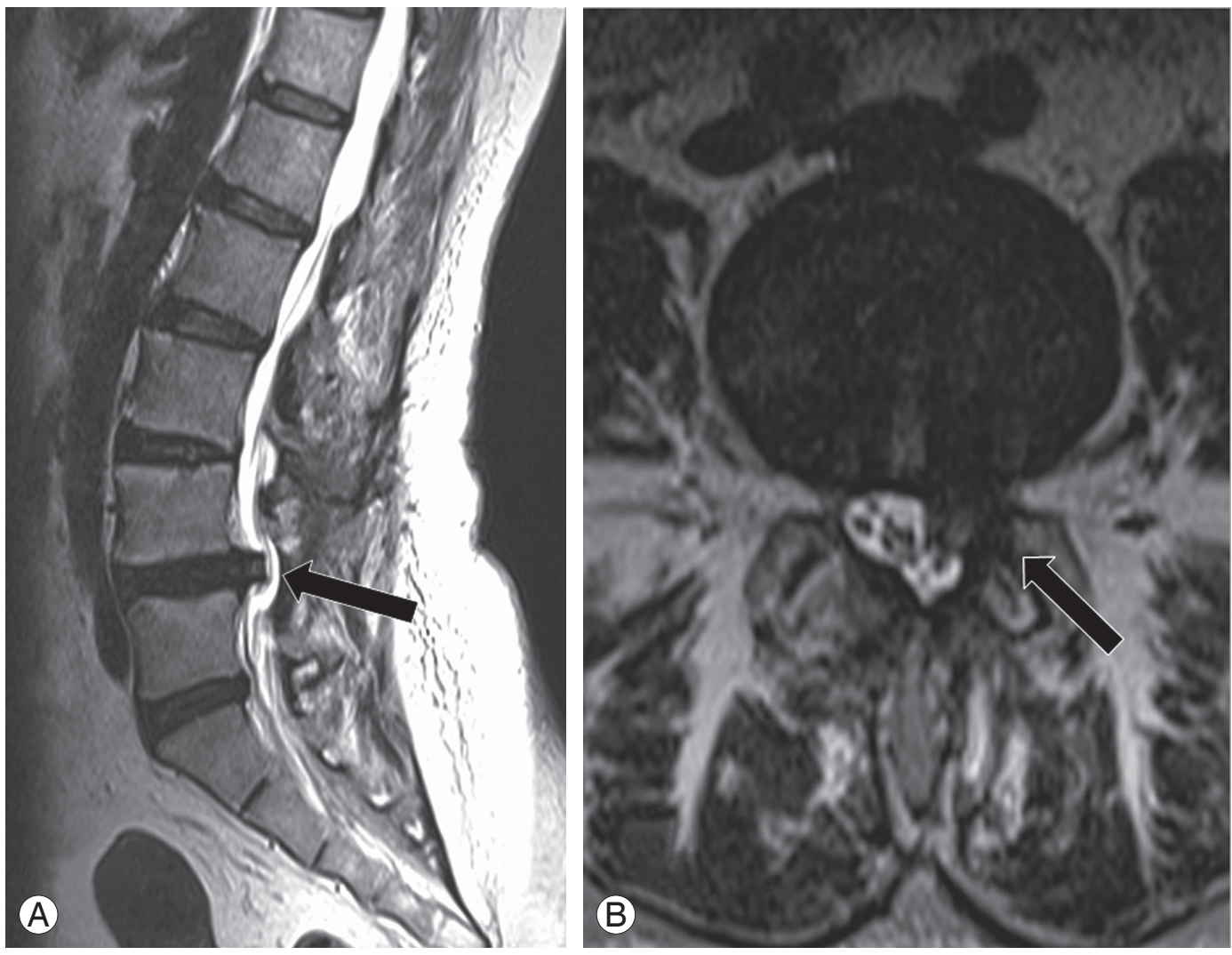

Fig. 2. A female patient, 50-year-old, with L3-L4, L4-L5 lateral recess stenosis underwent microsurgical posterior lumbar interbody fusion and decompression. Migration of the left cage to the spinal canal was observed at the $L 4-L 5$ level at the last (11-month) follow-up. Black arrows point to the migrated cage in (A) a sagittal view and (B) an axial view.

level fusions. The mean hospital stay was $2.5 \pm 0.5$ days (range, 2-4 days).

\section{Discussion}

LRS is usually accompanied by degenerative disc disease. Various surgical procedures have been used to surgically treat LRS: lumbar spinal fusions, such as posterolateral fusion, PLIF, translumbar interbody fusion, anterior lumbar interbody fusion, and decompressive surgery (including laminectomy and facetectomy. Traditional, open decompressive surgery and posterolateral fusion with additional posterior instrumentation have been widely used for LRS, but may cause musculoligamentous injuries $[12,20]$. Without accompanying posterior instrumentation, a posterolateral fusion cannot maintain the disc height and cannot contribute to the fusion $[8,9,12,21]$.

PLIF has been widely used as a surgical technique for spinal diseases, including spinal stenosis and degenerative disc disease. However, this technique, using an interbody cage without posterior stabilization, may lead to instability if a wide laminectomy and facetectomy are performed $[12,22]$. PLIF can also successfully restore disc height and anterior column alignment, as well as stabilize the disc space. Thus, the technique has the advantages of high fusion rates that lead to early stabilization, with the additional use of a pedicle screw fixation $[12,17,21,23]$. Posterior instrumentation, following PLIF, can hinder iatrogenic instability and may cause musculoligamentous injuries $[12,24]$. Denervation and atrophy may also result in the failed back syndrome, as reported in patients undergoing wide exposures [25]. However, PLIF without posterior instrumentation may result in postoperative complications, including instability, pseudoarthrosis, cage migration, and persistent back pain [12,17].

In observational studies using threaded fusion cages and other interbody fusion cages during PLIF, the interbody fusion rates and clinical symptom improvements are variable, with possible complications including migrated fusion cages, pseudoarthrosis, and exacerbated spinal 
instability [26]. Thus, solid fusion and complete decompression should be used, if possible, for the treatment of LRS, particularly to protect the posterior column [27]. Martin et al. [28] reported that, decompression and instrumentation were mainly used during PLIF. They also reported that iatrogenic injury of the lumbar spinal stability mechanism, including damaged anterior fiber rings and posterior longitudinal ligaments, increases the tendency of disc slippage and should be regarded as an important sign of poor postoperative fusion and incompletely improved symptoms. Ray [29] and Guo et al. [2] reported bone fusion rates of $96 \%$ (208 cases) and 100\% (48 cases), respectively, following PLIF using threaded fusion cages. Thus, this technique has become a successfully used surgical technique for the treatment of lumbar instability, compared with traditional lumbar fusion.

Recently, spinal microsurgery has been gaining popularity because of its advantages, which include less soft tissue damage, less intraoperative blood loss, faster postoperative pain relief, shorter hospital stays, and faster returns to daily life and work $[2,4,18,19]$. However, posterior instrumentation has not been used in the absence of lumbar spine instability, according to recent spinal microsurgery reports [2]. Lai et al. [30] noted that the posterior column structure integrity is aggravated by decompressive surgery, including total laminectomy and removal of the spinous process, and can lead to an adjacent segment instability. Hence, microscopy-guided resection of less than half of the medial facet, partial hemilaminotomy, and subsequent cage insertion through the resultant window can lead to stable posterior structures. The disadvantages of spinal microsurgery include a steep learning curve, incomplete spinal decompression, the need for special equipment, and complications, which include cage migration and pseudoarthrosis [2].

In the present study, although the increase in spinal canal diameter was significant, the increase in disc height was insufficient. It is hypothesized that this insufficient increase in disc height might be related to the patient position. Disc height decreases with the patient in the prone position and $90^{\circ}$ flexed hips; hence this could lead to a selection of a smaller size of the cage. In this study, the fusion rate $(85.2 \%)$ was lower and the migration rate $(5.8 \%)$ was higher when compared to the previously published literature $[1,7,8,12,16,20,24,26]$. An insertion of the small size cage causes instability and helps explains the lower fusion rate and higher migration rate. Thus, there seems to be a good argument that measurement of the disc height must be done with the patient in the prone position and extended hips. There are similar clinical results (Macnab criteria), operative times, intraoperative blood losses, and hospital stays, as have been previously reported $[1,2,7,8,12,14]$.

\section{Conclusions}

In summary, maintaining spinal stability, based on the rigid construct of a $360^{\circ}$ fusion mass, is important for the treatment of LRS [1]. Solid fusion, full decompression, and a limited approach are keys to successful surgery, but cage migration and pseudoarthrosis remain challenging complications to overcome when attempting to achieve an adequate lumbar interbody fusion. This clinical study demonstrated that the technological advances in PLIF microsurgery help the surgeon to achieve outcomes that are closer to the natural biomechanical properties of the lumbar spine than have been achieved with traditional approaches $[1,2,4,7,16,19,27,29]$. A minimally invasive approach, involving limited resection of the facet and lamina to facilitate cage insertion, prior to lumbar interbody fusion with additional posterior instrumentation, can lead to a stable spinal structure. Further studies, involving numerous centers and stable interbody cage designs will further the microsurgical treatment of lumbar LRS without the use of posterior instrumentation.

\section{Conflict of Interest}

No potential conflict of interest relevant to this article was reported.

\section{References}

1. Audat Z, Moutasem O, Yousef K, Mohammad B. Comparison of clinical and radiological results of posterolateral fusion, posterior lumbar interbody fusion and transforaminal lumbar interbody fusion techniques in the treatment of degenerative lumbar spine. Singapore Med J 2012;53:183-7.

2. Guo S, Sun J, Tang G. Clinical study of bilateral decompression via vertebral lamina fenestration for lumbar interbody fusion in the treatment of lower lumbar instability. Exp Ther Med 2013;5:922-6.

3. Nydegger A, Bruhlmann P, Steurer J. Lumbar spinal 
stenosis: diagnosis and conservative treatment. Praxis (Bern 1994) 2013;102:391-8.

4. Oppenheimer JH, DeCastro I, McDonnell DE. Minimally invasive spine technology and minimally invasive spine surgery: a historical review. Neurosurg Focus 2009;27:E9.

5. Lane WA. Case of spondylolisthesis associated with progressive paraplegia: laminectomy. Lancet 1893; 141:991-2.

6. Young S, Veerapen R, O'Laoire SA. Relief of lumbar canal stenosis using multilevel subarticular fenestrations as an alternative to wide laminectomy: preliminary report. Neurosurgery 1988;23:628-33.

7. Wu WJ, Liang Y, Zhang XK, Cao P, Zheng T. Complications and clinical outcomes of minimally invasive transforaminal lumbar interbody fusion for the treatment of one- or two-level degenerative disc diseases of the lumbar spine in patients older than 65 years. Chin Med J (Engl) 2012;125:2505-10.

8. Ha KY, Na KH, Shin JH, Kim KW. Comparison of posterolateral fusion with and without additional posterior lumbar interbody fusion for degenerative lumbar spondylolisthesis. J Spinal Disord Tech 2008; 21:229-34.

9. Bridwell KH, Sedgewick TA, O’Brien MF, Lenke LG, Baldus $C$. The role of fusion and instrumentation in the treatment of degenerative spondylolisthesis with spinal stenosis. J Spinal Disord 1993;6:461-72.

10. Feffer HL, Wiesel SW, Cuckler JM, Rothman RH. Degenerative spondylolisthesis: to fuse or not to fuse. Spine (Phila Pa 1976) 1985;10:287-9.

11. Cloward RB. The treatment of ruptured lumbar intervertebral discs by vertebral body fusion. I. indications, operative technique, after care. J Neurosurg 1953;10:154-68.

12. Seong JH, Lee JW, Kwon KY, Rhee JJ, Hur JW, Lee HK. Comparative study of posterior lumbar interbody fusion via unilateral and bilateral approaches in patients with unilateral leg symptoms. J Korean Neurosurg Soc 2011;50:363-9.

13. Bagby GW. Arthrodesis by the distraction-compression method using a stainless steel implant. Orthopedics 1988;11:931-4.

14. Fogel GR, Toohey JS, Neidre A, Brantigan JW. Is one cage enough in posterior lumbar interbody fusion: a comparison of unilateral single cage interbody fusion to bilateral cages. J Spinal Disord Tech 2007;20:60-5.
15. Styf JR, Willen J. The effects of external compression by three different retractors on pressure in the erector spine muscles during and after posterior lumbar spine surgery in humans. Spine (Phila Pa 1976) 1998; 23:354-8.

16. Yao N, Wang W, Liu Y. Percutaneous endoscopic lumbar discectomy and interbody fusion with BTwin expandable spinal spacer. Arch Orthop Trauma Surg 2011;131:791-6.

17. Chen L, Yang H, Tang T. Cage migration in spondylolisthesis treated with posterior lumbar interbody fusion using BAK cages. Spine (Phila Pa 1976) 2005; 30:2171-5.

18. Logroscino CA, Proietti L, Pola E, Scaramuzzo L, Tamburrelli FC. A minimally invasive posterior lumbar interbody fusion for degenerative lumbar spine instabilities. Eur Spine J 2011;20 Suppl 1:S41-5.

19. Guiot BH, Khoo LT, Fessler RG. A minimally invasive technique for decompression of the lumbar spine. Spine (Phila Pa 1976) 2002;27:432-8.

20. Axelsson P, Johnsson R, Stromqvist B, Arvidsson M, Herrlin K. Posterolateral lumbar fusion: outcome of 71 consecutive operations after 4 (2-7) years. Acta Orthop Scand 1994;65:309-14.

21. Chen L, Tang T, Yang H. Complications associated with posterior lumbar interbody fusion using Bagby and Kuslich method for treatment of spondylolisthesis. Chin Med J (Engl) 2003;116:99-103.

22. Lin PM. Posterior lumbar interbody fusion (PLIF): past, present, and future. Clin Neurosurg 2000;47: 470-82.

23. Hitchon PW, Goel V, Rogge T, Dooris A, Drake J, Torner J. Spinal stability with anterior or posterior ray threaded fusion cages. J Neurosurg 2000;93:1028.

24. Foley KT, Gupta SK. Percutaneous pedicle screw fixation of the lumbar spine: preliminary clinical results. J Neurosurg 2002;97:7-12.

25. Sihvonen T, Herno A, Paljarvi L, Airaksinen O, Partanen J, Tapaninaho A. Local denervation atrophy of paraspinal muscles in postoperative failed back syndrome. Spine (Phila Pa 1976) 1993;18:575-81.

26. Rodgers WB, Gerber EJ, Patterson J. Intraoperative and early postoperative complications in extreme lateral interbody fusion: an analysis of 600 cases. Spine (Phila Pa 1976) 2011;36:26-32.

27. Shunwu F, Xing Z, Fengdong Z, Xiangqian F. Mini- 
mally invasive transforaminal lumbar interbody fusion for the treatment of degenerative lumbar diseases. Spine (Phila Pa 1976) 2010;35:1615-20.

28. Martin BI, Mirza SK, Comstock BA, Gray DT, Kreuter W, Deyo RA. Are lumbar spine reoperation rates falling with greater use of fusion surgery and new surgical technology? Spine (Phila Pa 1976) 2007; 32:2119-26.
29. Ray CD. Threaded fusion cages for lumbar interbody fusions: an economic comparison with 360 degrees fusions. Spine (Phila Pa 1976) 1997;22:681-5.

30. Lai PL, Chen LH, Niu CC, Fu TS, Chen WJ. Relation between laminectomy and development of adjacent segment instability after lumbar fusion with pedicle fixation. Spine (Phila Pa 1976) 2004;29:2527-32. 